\title{
El entrenamiento en juicios metamnemónicos y su implicación en el rendimiento académico
}

\section{Trainings of the metamneconics judgments and involvement in the academic achievement}

\author{
María José Pérez-Fabello, Jorge Soto-Carballo \\ Departamento de Análisis e Intervención Psicosocioeducativa, Universidade de Vigo, España
}

\begin{abstract}
Resumen
Este estudio se desarrolló durante el transcurso de la docencia de la asignatura de Psicología del Arte en el Grado de Bellas Artes (segundo curso de la Facultad de Bellas Artes de la Universidad de Vigo). El objetivo de este trabajo era averiguar la relación entre distintas variables de la metamemoria (Facilidad de Aprendizaje, Juicio de Aprendizaje y Juicio de Confianza) a medida que iba transcurriendo el curso, y la relación entre estas variables y el rendimiento académico y la capacidad de imagen en una muestra de 90 estudiantes. De los resultados cabe destacar que el entrenamiento en la valoración de los juicios metamnemónicos incrementó la correlación entre los juicios a medida que transcurría el curso, generando una tendencia en la respuesta que creemos es fruto de un aprendizaje. Además, se puso de manifiesto correlaciones altas y significativas entre los distintos juicios metamnemónicos y la evaluación final en la asignatura.
\end{abstract}

Palabras Clave: imagen mental, juicios metamnemónicos, rendimiento académico.

\begin{abstract}
This study was developed during the course of the teaching of the subject of Psychology of Art in Fine Arts degree (second year of the Faculty of Fine Arts at the University of Vigo). The aim of this study was to determine the relationship between different variables metamemory (Ease of Learning, Judgment of Learning and Confidence Level) during the course, and the relationship between these variables and academic performance and capacity image on a sample of 90 students. From the results it should be noted that training in the assessment of metamnemonic judgments increased the correlation between judgments as they passed the course, generating a trend in the response we believe is the result of learning. Furthermore, showed high and significant correlations between metamnemonic judgments and final evaluation in the subject.
\end{abstract}

Keywords: mental imagery, metamnemonic judgments, academic achievement.

\section{Introducción}

El actual paradigma cognitivo propone modelos teóricos que delimitan el funcionamiento e intervención en la mejora de los procesos cognitivos y del aprendizaje académico. En este sentido cabe destacar los estudios centrados en las estrategias de aprendizaje dentro del proceso de aprendizaje (Beltrán, 1993; Li, 2000; Monereo, 1997; Mayor, Suengas, González-Marqués, 1993; Pérez Cabaní, 1997; Ruiz, 2008). Estos estudios ponen de manifiesto la importancia de tomar en consideración el conocimiento estratégico del alumno que aprende, o lo que es lo mismo, la metacognición. De la Fuente y Justicia (2003) define la metacognición como el grado de conocimiento que la persona tiene de sí misma (sus posibilidades, limitaciones, etc.), sobre los requerimientos de la tarea que lleva a cabo (pasos que incluye, dificultades, etc.) y sobre el propio proceso de aprendizaje (cómo está aprendiendo, los errores que comete, etc.). La metacognición supone que la persona toma conciencia, reflexiona, regula y se responsabiliza de su propio proceso de aprendizaje.

Un campo de estudio más restringido, dentro del ámbito de la metacognición, es la metamemoria, que se ocupa de los juicios que hacen las personas acerca del conocimiento de cierta información y del modo en que esos juicios pueden afectar al comportamiento global del individuo $o$ a las estrategias particulares de procesamiento (Ruíz, 2008).

Nelson y Narens (1990) distinguieron entre las actividades de la metamemoria prospectiva que se realizan durante la adquisición de información, que han denominado juicios de aprendizaje (JOL “judgment learning” o JOK “judgment of knowledge”) y aquellas actividades de metamemoria prospectiva que se realizan en el momento de la recuperación de la información, a las que denominaron impresión de saber (FOK "feeling of knowing").

Ruíz en 2008 señala que los juicios metamnemónicos se pueden realizar en cuatro momentos diferentes del proceso de aprendizaje: (a) ante la presentación del material, cuando todavía no se ha producido un intento voluntario de aprendizaje, en este momento se puede estimar la mayor o menor facilidad o dificultad en el aprendizaje (Juicios de Facilidad de Aprendizaje) (b) durante el aprendizaje, y después de éste, pero antes de una prueba de recuperación, se puede valorar el nivel del conocimiento que se ha alcanzado (Juicios de Aprendizaje) (c) durante la prueba podemos estimar la el éxito esperado en otra prueba de igual o distinto formato (Sensación de Saber); y (d) una vez recuperado el material, durante la evaluación, se puede especificar el 
grado de seguridad en que la respuesta es correcta (Juicios de Confianza).

Utilizando estos juicios metamnemónicos cabe destacar los estudios pioneros de Ameijide y Campos (2013a, 2013b) aplicados a la mnemotecnia de la palabra clave con personas mayores. Estos trabajos miden la viveza de imagen de los participantes y su influencia en la facilidad de aprendizaje y en el juicio de aprendizaje utilizando distintas estrategias de presentación y materiales diferentes. Los resultados pusieron de manifiesto que los participantes altos en viveza de imagen obtuvieron puntuaciones significativamente mayores tanto en la facilidad de aprendizaje como en los juicios de aprendizaje. Siguiendo la misma línea de trabajo, Campos y Ameijide (2014) estudiaron si el tipo de estrategia utilizada en el aprendizaje influía en el recuerdo y en los juicios metamnemónicos. Estos autores concluyeron que el método de mnemotecnia de la palabra clave reforzada con dibujos fue superior al método habitual de aprendizaje en el recuerdo y en todos los juicios metamnemónicos estudiados.

En una línea de trabajo centrada en estudiantes universitarios Escanero, Soria, Escanero y Guerra (2013) con estudiantes de medicina y Martinez-Fernández, Tubau, Gilera, Rabanaque y Sanchez (2008) con estudiantes de Psicología, se evidencia la importancia de las estrategias metacognitivas en la mejora del rendimiento académico y la resolución de problemas de insight, respectivamente.

A la luz de los modelos y evidencias de la Psicología de la Educación parece necesario tomar decisiones relevantes en cuanto al diseño y desarrollo de los procesos enseñanza-aprendizaje del aula en el contexto universitario. Partiendo de este contexto, se realiza este estudio (ya iniciado en un trabajo preliminar, ver Pérez-Fabello, 2011) con la intención de averiguar la relación entre distintas variables de la metamemoria a medida que iba transcurriendo el curso y la relación entre estas variables, el rendimiento académico y la capacidad de imagen.

\section{Método}

\section{Participantes}

La muestra estaba formada por 90 estudiantes de la Facultad de Bellas Artes de la Universidad de Vigo (23 hombres y 67 mujeres). La media de edad fue de 19,91 años (SD = 1.52, rango 18 a 24 años). Todos participaron voluntariamente.

\section{Material y Procedimiento}

Los juicios metamnemónicos que se emplearon fueron los siguientes:

1.- Facilidad de Aprendizaje (EOL, Ease of Learning). En este juicio se valora la probabilidad de aprender contenidos en una escala de 100 puntos que va de 0 , muy difícil de aprender a 100, muy fácil de aprender. Las puntuaciones podían oscilar de $0,20,40,60,80,100$, tiene que rodear con un círculo lo que proceda.
2.- Juicio de Aprendizaje (JOL, Judgment of Learning). En este juicio se valora la probabilidad de recordar contenidos en una escala de 100 puntos ( $(0=$ estoy completamente seguro que no los recordaré, y $100=$ estoy seguro que los recordaré). Las puntuaciones pueden ser: 0, 20, 40, 60, 80, 100.

3.- Juicio de Confianza (CL, Confidence Level). En este juicio se valora el nivel de confianza que se tiene de que las respuestas que dimos son correctas. Se puntúa en una escala de 1 a 5 la seguridad de que la respuesta es correcta. El 1 indica poca seguridad y el 5 indica mucha seguridad.

A los participantes se les pasaron las siguientes pruebas de imágenes mentales:

Mental Rotation Test (MRT; Vandenberg \& Kuse, 1978). Este test tiene 10 items en los que se compara una figura geométrica tridimensional, figura criterio, con otras cuatro figuras, de las cuales dos opciones son correctas y dos incorrectas. La tarea de los participantes requiere rotar las figuras para ver si son iguales a la figura criterio o están en espejo. La puntuación máxima es 20. Los participantes tienen un tiempo máximo de 3 minutos para terminar el test.

The Measure of the Ability to Form Spatial Mental Images (MASMI; Campos, 2009). La prueba consiste en un cubo desplegado que los participantes tienen que volver a montar antes de responder a 23 preguntas en un límite de tiempo de 10 minutos. Cada pregunta tiene cuatro opciones, dos son correctas y dos incorrectas. Se calcula la puntuación total de la prueba mediante la suma de las opciones correctas y restando las incorrectas; por lo tanto, la puntuación total podría oscilar entre - 46 a 46.

Utilizamos la versión española (Pérez-Fabello \& Campos, 2004) del Gordon Test of Visual Imagery Control (TVIC; Richardson, 1969). El test mide la capacidad de control de imagen que tienen los participantes. Consta de 12 ítems a través de los cuales se pide a los participantes que representen mentalmente un coche en distintas posiciones. La respuesta "sí” se puntúa con dos puntos, la respuesta "inseguro" se puntúa con un punto, y la respuesta “no”, se puntúa con cero puntos. La puntuación puede oscilar entre 0 y 24.

Por último utilizamos la versión española (Campos, González, \& Amor, 2002) del Vividness of Visual Imagery Questionnaire (VVIQ) (Marks, 1973). El test mide la capacidad de los participantes para formar imágenes vivas. Consta de 16 ítems que se responden dos veces, la primera con los ojos abiertos y la segunda con ellos cerrados. Altas puntuaciones en el test indican baja viveza de imagen y viceversa.

El Rendimiento Académico se midió a través de una prueba tipo test de 30 preguntas con 4 alternativas. El examen fue diseñado de modo que hubiese el mismo número de preguntas por cada uno de los nueve temas e igual grado de dificultad. Se anotaron los aciertos por tema y el total.

Durante el curso, después de la explicación de cada tema los alumnos tuvieron que puntuar la Facilidad de Aprendizaje (EOL) de cada uno de los temas. Una vez que se presentaron todos los temas y se estudiaron para el examen se les entregó el Juicio de Aprendizaje (JOL) de cada uno de los temas, esto se llevó a cabo justo antes del 
examen. Finalmente, cuando realizaron el examen y después de que contestaron a cada pregunta cubrieron el Juicio de Confianza (CL).

Las pruebas de imagen se pasaron durante las clases prácticas, en grupos pequeños de alrededor de 20 participantes.

\section{Resultados}

Se ha realizado una Correlación de Pearson con cada uno de los Juicios metamnemónicos en cada uno de los temas con el fin de ver cómo se comportaba el alumnado a medida que iban realizando los juicios. La Facilidad de Aprendizaje de cada uno de los temas (ver Tabla 1) se puede apreciar mayor correlación a medida que se desarrolla el curso y que se van realizando juicios

Tabla 1.

Matriz de Intercorrelación de Pearson de Facilidad de Aprendizaje por Temas

\begin{tabular}{llllllll}
\hline FA & FA1 & FA2 & FA3 & FA4 & FA5 & FA6 & FA7 \\
\hline FA2 & .10 & & & & & & \\
FA3 & .25 & $.48^{* *}$ & & & & & \\
FA4 & $.29 *$ & $.43^{* *}$ & .17 & & & & \\
FA5 & .08 & $.44^{* *}$ & $.43^{* *}$ & $.37 * *$ & & & \\
FA6 & .24 & $.49^{* *}$ & $.41^{* *}$ & $.30^{*}$ & $.65^{* *}$ & & \\
FA7 & .13 & .25 & .17 & $.29^{*}$ & $.40^{* *}$ & .24 & \\
FA8 & .19 & $.42^{* *}$ & .19 & $.42^{* *}$ & $.45^{* *}$ & $.46^{* *}$ & $.66^{* *}$ \\
\hline$* p<.05 .{ }^{* *} p<.01$. & & & & &
\end{tabular}

También se puede apreciar esa mayor correlación a medida que van transcurriendo los temas en los Juicios de Aprendizaje (ver Tabla 2), aunque aquí las correlaciones ya empiezan a ser significativas desde el principio.

Tabla2.

Matriz de Intercorrelación de Pearson de Juicios de Aprendizaje por Temas

\begin{tabular}{llllllll}
\hline JA & JA1 & JA2 & JA3 & JA4 & JA5 & JA6 & JA7 \\
\hline JA2 & $.39^{* *}$ & & & & & & \\
JA3 & $.25^{*}$ & $.50^{* *}$ & & & & & \\
JA4 & $.27^{*}$ & $.42^{* *}$ & $.54^{* *}$ & & & & \\
JA5 & .12 & $.34^{* *}$ & $.54^{* *}$ & $.50^{* *}$ & & & \\
JA6 & $.29^{* *}$ & $.32^{* *}$ & $.46^{* *}$ & $.29 * *$ & $.42^{* *}$ & & \\
JA7 & $.25^{*}$ & $.25^{*}$ & $.45^{* *}$ & $.28^{* *}$ & $.39^{* *}$ & $.51^{* *}$ & \\
JA8 & $.23^{*}$ & .20 & $.38^{* *}$ & $.34^{* *}$ & $.29^{* *}$ & $.42^{* *}$ & $.45^{* *}$ \\
\hline${ }^{*} p<.05 .{ }^{* *}<<.01$. & & & & &
\end{tabular}

De forma más clara y contundente se puede apreciar en los Juicios de Confianza (ver Tabla 3). Las correlaciones de los Juicios de Confianza en los últimos temas son más altas y son significativas desde el principio al .01.

Las correlaciones entre los test de imagen son altas y significativas (ver Tabla 4), especialmente los dos test de rendimiento entre sí, MRT y MASMI y los cuestinarios entre sí, GORDON y VVIQ.
Tabla 3.

Matriz de Intercorrelación de Pearson de Juicios de Confianza por Temas

\begin{tabular}{llllllll}
\hline JC & JC1 & JC2 & JC3 & JC4 & JC5 & JC6 & JC7 \\
\hline JC2 & $.43^{* *}$ & & & & & & \\
JC3 & $.46^{* *}$ & $.36^{* *}$ & & & & & \\
JC4 & $.44^{* *}$ & $.36^{* *}$ & $.46^{* *}$ & & & & \\
JC5 & $.50^{* *}$ & $.48^{* *}$ & $.42^{* *}$ & $.58^{* *}$ & & & \\
JC6 & $.41^{* *}$ & $.33^{* *}$ & $.52^{* *}$ & $.53^{* *}$ & $.63^{* *}$ & & \\
JC7 & $.52^{* *}$ & $.38^{* *}$ & $.55^{* *}$ & $.62^{* *}$ & $.59^{* *}$ & $.53^{* *}$ & \\
JC8 & $.45^{* *}$ & $.36^{* *}$ & $.47^{* *}$ & $.56^{* *}$ & $.56^{* *}$ & $.51^{* *}$ & $.60^{* *}$ \\
\hline
\end{tabular}

$* * p<.01$.

Tabla 4.

Matriz de Intercorrelación de Pearson de Test de Imagen

\begin{tabular}{llll}
\hline & MRT & MASMI & GORDON \\
\hline MASMI & $.45^{* *}$ & & \\
GORDON & $.25 *$ & .15 & \\
VVIQ & .09 & .19 & $-.32 * *$ \\
\hline${ }^{*} p<.05 .{ }^{* *} p<.01$. & &
\end{tabular}

En las correlaciones entre los test de imagen y los juicios metacognitivos se encontró correlación entre el GORDON y el juicio de Facilidad de Aprendizaje total $(.70, p<.01)$ y el GORDON y el Juicio de Aprendizaje total $(.25, p<.05)$.

Por último hay que destacar la correlación que se encontró entre los juicios metamnemónicos y el rendimiento en la asignatura. Así, se ha encontrado que la Facilidad de Aprendizaje Total (.57, $p<.01)$, el Juicio de Aprendizaje Total $(.29, p<.01)$ y el Juicio de Confianza Total (.54, $p<.001)$ correlacionaron significativamente con la puntuación en el examen.

Las correlaciones altas y significativas a medida que los participantes realizan los juicios parecen poner de manifiesto que el entrenamiento, a medida que los participantes van emitiendo juicios, genera una tendencia y aumenta su seguridad en el juicio. La correlación entre el GORDON Test y la Facilidad y el Juicio de Aprendizaje corrobora los resultados de estudios previos en los que se comprueba la influencia de la capacidad de imagen en distintos juicios de aprendizaje (Ameijide y Campos (2013a, 2013b). Del mismo modo, la correlación entre los juicios metamnemónicos y el rendimiento en la asignatura, parece poner de nuevo de manifiesto la importancia de la autorreflexión en el proceso de enseñanza-aprendizaje (Escanero, Soria, Escanero y Guerra, 2013; Martinez-Fernández, Tubau, Gilera, Rabanaque y Sanchez, 2008).

La creación del Espacio Europeo de Educación Superior (EEES) conlleva el cambio del paradigma de la enseñanza superior que pasa de estar centrado en el profesor a estarlo en el alumno. Ello implica dotar al alumno de las herramientas más idóneas para ello y, especialmente, del conocimiento de sus propios procesos 
de aprendizaje para poder elegir siempre el más óptimo $\mathrm{y}$, en caso de que así no sea, poder rectificar y cambiarlo.

\section{Referencias}

Ameijide, L., y Campos, A. (2013a). Percepción de facilidad de aprender (EOL) listas de palabras mediante la mnemotecnia de la palabra clave con dibujos. En R. González Cabanach, R. Fernández Cervantes, F. Fariña Rivera, M. Vilariño Vázquez, y C. Freire Rodríguez (Eds). Psicología y salud I. Educación, aprendizaje y salud (pp. 103-111). A Coruña: GEU Editorial.

Ameijide, L., y Campos, A. (2013b). Influencia de la viveza de imagen en los juicios de aprendizaje (EOL) utilizando la mnemotrecnia de la palabra clave reforzada con dibujos. En R. González Cabanach, R. Fernández Cervantes, F. Fariña Rivera, M. Vilariño Vázquez, y C. Freire Rodríguez (Eds). Psicología y salud I. Educación, aprendizaje y salud (pp. 113-120). A Coruña: GEU Editorial.

Beltrán, J. A. (1993). Procesos, estrategias y técnicas de aprendizaje. Madrid: Síntesis, S. A.

Campos, A. (2009). Spatial imagery: A new measure of the visualization factor. Imagination, Cognition and Personality, 29, 31-39.

Campos, A., y Ameijide, L. (2014). Mnemotecnia de la palabra clave con dibujos y juicios metamnemónicos de personas mayores. Revista Iberoamericana de Psicología y Salud, 5, 23-38.

Campos, A., González, M. A., y Amor, A. (2002). The Spanish version of the Vividness of Visual Imagery Questionnaire: Factor structure and internal consistency reliability (VVIQ). Psychological Reports, 90, 503-506.

De la Fuente, J., y Justicia, F. (2003). Regulación de la enseñanza para la autorregulación del aprendizaje. Aula Abierta, 82, 161-171.

Escanero, J. F., Soria, M., Escanero, M. L., y Guerra, M. (2013). Estilos, metacognición y estrategias de aprendizaje en estudiantes de medicina. Una propuesta para la mejora de la enseñanza/aprendizaje. Revista de Farmacología de Chile, 6, 39-47.

Li, X. (2000). Differently adapatative pupils projets responses to the open-ended task: A research on the mechanism of self-regulation. Psychological Science China, 23, 664-668.

Marks, D. (1973). Visual imagery differences in the recall of pictures. British Journal of Psychology, 64, $17-24$.

Martinez-Fernández, R., Tubau, E., Gilera, Ll., Rabanaque, S., y Sanchez, E. (2008). Utilidad de distintas ayudas en la resolución de un problema de insight y su relación con las estrategias metacognitivas. Anales de Psicología, 24, 16-24.

Mayor, J., Suengas, A., y González-Marqués, J. G. (1993). Estrategias metacognitiva. Aprender a aprender y aprender a pensar. Madrid: Síntesis.

Monereo, C. (1997). Estrategias de aprendizaje. Barcelona: Visor-UOC.

Nelson, T. O., y Narens, L. (1990). Metamemory: A theoretical framework and new findings. En G. H.
Bower (Coord.). The psychology of learning and motivation: Advances in research and theory (Vol. 22, pp. 227-259). San Diego, CA: Academic Press.

Pérez Cabaní, M. L. (1997). La enseñanza y el aprendizaje de estrategias desde el currículum. Barcelona: Horsori.

Pérez-Fabello, M. J. (2011). Los juicios metacognitivos y el rendimiento académico: Un estudio preliminar. Actas del XI Congresso Internacional Galego-Português de Psicopedagogía (pp.4155-4160). A Coruña: Universidade da Coruña. ISBN: 1138-1663.

Pérez-Fabello, M. J., y Campos, A. (2004). Factor structure and internal consistency of the Spanish version of the Gordon Test of Visual Imagery Control. Psychological Reports, 94, 761-766.

Richardson, A. (1969). Mental imagery. Routledge \& Kagan Paul.

Ruiz, M. (2008). Las caras de la memoria. Madrid: Pearson Educación, S.A.

Vandenberg, S. G., y Kuse, A. R. (1978). Mental rotations, a group test of three-dimensional spatial visualization. Perceptual and Motor Skills, 47, 599-604. 